\title{
Controlled On-Chip Single-Photon Transfer Using Photonic Crystal Coupled-Cavity Waveguides
}

\author{
Hubert Pascal Seigneur, ${ }^{1}$ Matthew Weed, ${ }^{1}$ Michael Niklaus Leuenberger, ${ }^{2,3}$ \\ and Winston Vaughan Schoenfeld ${ }^{1}$
}

${ }^{1}$ CREOL, The College of Optics and Photonics, University of Central Florida, Orlando, FL 32826, USA

${ }^{2}$ NanoScience Technology Center, University of Central Florida, Orlando, FL 32816, USA

${ }^{3}$ Department of Physics, University of Central Florida, P.O. Box 162385, Orlando, FL 32816, USA

Correspondence should be addressed to Hubert Pascal Seigneur, seigneur@creol.ucf.edu

Received 15 June 2010; Revised 21 September 2010; Accepted 1 November 2010

Academic Editor: Martin Cryan

Copyright (C) 2011 Hubert Pascal Seigneur et al. This is an open access article distributed under the Creative Commons Attribution License, which permits unrestricted use, distribution, and reproduction in any medium, provided the original work is properly cited.

To the end of realizing a quantum network on-chip, single photons must be guided consistently to their proper destination both on demand and without alteration to the information they carry. Coupled cavity waveguides are anticipated to play a significant role in this regard for two important reasons. First, these structures can easily be included within fully quantum-mechanical models using the phenomenological description of the tight-binding Hamiltonian, which is simply written down in the basis of creation and annihilation operators that move photons from one quasimode to another. This allows for a deeper understanding of the underlying physics and the identification and characterization of features that are truly critical to the behavior of the quantum network using only a few parameters. Second, their unique dispersive properties together with the careful engineering of the dynamic coupling between nearest neighbor cavities provide the necessary control for high-efficiency single-photon on-chip transfer. In this publication, we report transfer efficiencies in the upwards of $93 \%$ with respect to a fully quantum-mechanical approach and unprecedented $77 \%$ in terms of transferring the energy density contained in a classical quasibound mode from one cavity to another.

\section{Introduction}

In order to obtain an efficient quantum computing architecture, the general consensus is that various implementations of the qubit should be combined. This calls on the one hand for stationary qubits that are good for storage, such as atoms to be used at quantum network nodes, and on the other hand for flying qubits that have desirable properties for travel, such as photons to be used as quantum interconnects. Moreover, the storage qubits can map their quantum state onto the traveling qubits and vice versa by means of coherent interfaces [1-3]. With the intention of realizing an efficient quantum computing architecture, this composite qubit approach to a quantum technology has been proposed for ion trap qubits [4] and also for neutral atoms [5]. We, in addition, have proposed a similar approach in connection with semiconductor-based artificial atoms or quantum dots
[6]. Regardless of choice, these various implementations of the composite qubit architecture are only possible if single photons are able to be guided from one node to another with both high efficiency and fidelity.

Recently, the on-chip generation and transfer of microwave single photons have been demonstrated in connection with superconducting qubits via transmission line cavities [7-10]. In addition, the generation and transfer of single photons on photonic crystal chips using a $25 \mu \mathrm{m}$ long defect waveguide structure has been studied by England et al. showing $12 \%$ transfer efficiency with quantum dots inside the nodes and $49 \%$ transfer efficiency without [11]. In this publication, we investigate the use of a photonic crystal coupled-cavity waveguide (CCW) for the generation and transfer of single photons on-chip. Such approach to an on-chip quantum network present several advantages. For example, since the photons we use have a wavelength of 


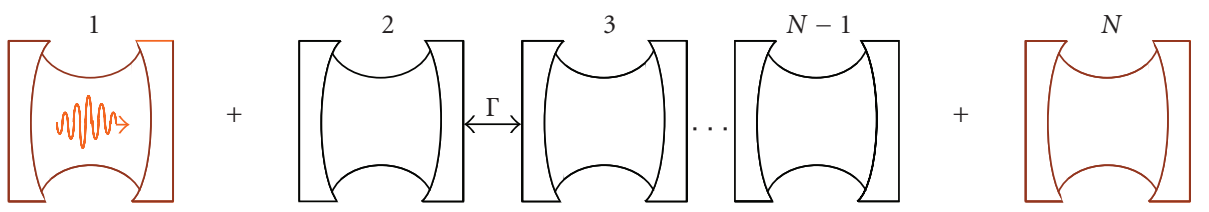

FIGURE 1: Two high-Q cavities connected via a coupled-cavity waveguide.

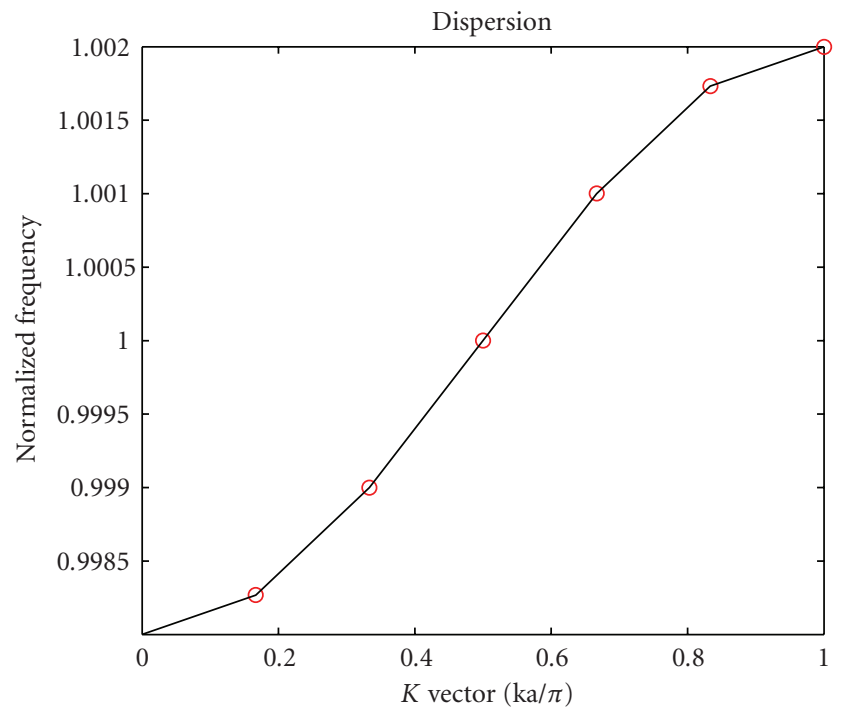

FIgURe 2: Dispersion of a 12 coupled-cavities waveguide.

around $1 \mu \mathrm{m}$ (IR) as opposed to $1 \mathrm{~cm}$ or more (microwave), the surface area of a quantum network in any optical system would be a great deal smaller and thus much more suitable for on-chip integration. In addition, the transfer of microwave photons was shown to take few hundred nanoseconds, whereas the transfer of optical photons inside the photonic crystal chip is anticipated to take no more than few tens of picoseconds.

Furthermore, CCWs offer a truly unique and sophisticated control over the transport of single photons [12]. Because modes of CCWs resemble those of the high- $Q$ cavity modes and possess the same field symmetries, these devices can be used to make bends with no reflection. In addition, they can dramatically slow down optical waves, and because of their versatile dispersion properties (both positive and negative dispersion are achievable), they allow for a great deal of control over a single-photon pulse propagation. Moreover, since each mode is strongly localized, the guided mode is composed of a linear combination of these individual bound modes. This renders the propagating mode easy to model quantum-mechanically [13], therefore allowing for their guiding behavior to be optimized with respect to maximized fidelity of quantum operations inside the quantum network. And, once their guiding behavior has been characterized with a few parameters, namely, the coupling coefficient $\kappa$ and resonant frequency $\omega_{c}$, their physical structure can be constructed to emulate their intended behavior.

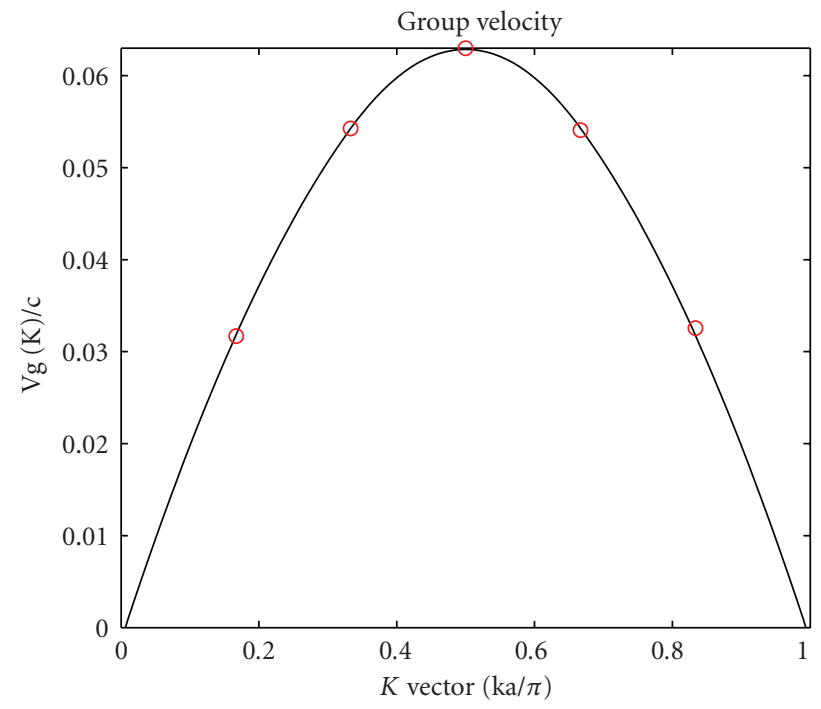

Figure 3: Group Velocity.

The paper is organized as follows. The modeling of the on-chip single-photon transfer dynamics is described in Section 2. Included in that section are the single-photon pulse propagation characteristics, the engineering of the source/target cavity to waveguide dynamic coupling as well as resulting transfer efficiencies. A physical implementation of a CCW based on a photonic crystal structure for the purpose of transferring single photon on-chip is presented in Section 3. An analysis and discussion of the performance of CCWs is provided in Section 4, followed by a final summary and conclusions in Section 5.

\section{Modeling On-Chip Single-Photon Transfer Dynamics}

The device under investigation is depicted in Figure 1. It consists of two cavities, cavity 1 and cavity $N$, linked to one another by means of a CCW, consisting of cavity 2 through cavity $N-1$. In this specific example, $N$ is 12 . The coefficient $\Gamma$ describes the weak coupling between the cavities forming the waveguide.

A general Hamiltonian for this system is derived from the tight-binding Hamiltonian and it is shown in (1). $\hat{\mathbf{a}}_{j}^{\dagger}$ and $\hat{\mathbf{a}}_{j}$ are the creation and the annihilation for the field in the $j$ th cavity, $\omega_{c}$ is the resonant cavities frequency, $V_{C_{1}-W}(t)$ is the time-dependent interaction between the first cavity and the 
waveguide, and $V_{W-C_{N}}(t)$ is the time-dependent interaction between the waveguide and the last cavity

$$
\begin{aligned}
\hat{\mathbf{H}}= & \sum_{j} \hbar \omega_{c} \hat{\mathbf{a}}_{j}^{\dagger} \hat{\mathbf{a}}_{j}+\sum_{2}^{N-2} \Gamma\left(\hat{\mathbf{a}}_{j}^{\dagger} \hat{\mathbf{a}}_{j+1}+\hat{\mathbf{a}}_{j+1}^{\dagger} \hat{\mathbf{a}}_{j}\right) \\
& +V_{C_{1}-W}(t)+V_{W-C_{N}}(t) .
\end{aligned}
$$

Our key concern in this publication is the engineering of the interaction between cavities and the waveguide structure $V_{C_{1}-W}(t)$ and $V_{W-C_{N}}(t)$. First, we consider the waveguide by itself and design its characteristics. Then, both the cavities and the waveguide are considered, and the system is engineered such that the photon is unloaded into the waveguide and transferred to its destination with minimum loss.

2.1. Waveguide. First, it is useful to consider a CCW by itself. The Hamiltonian that can describe such a waveguide is exactly the tight-binding Hamiltonian in

$$
\hat{\mathbf{H}}_{\mathrm{TB}}=\sum_{j} \hbar \omega_{c} \hat{\mathbf{a}}_{j}^{\dagger} \hat{\mathbf{a}}_{j}+\sum_{j} \Gamma\left(\hat{\mathbf{a}}_{j}^{\dagger} \widehat{\mathbf{a}}_{j+1}+\hat{\mathbf{a}}_{j+1}^{\dagger} \widehat{\mathbf{a}}_{j}\right),
$$

In the Wannier representation, essentially a real-space picture of localized orbitals, this Hamiltonian matrix is written as

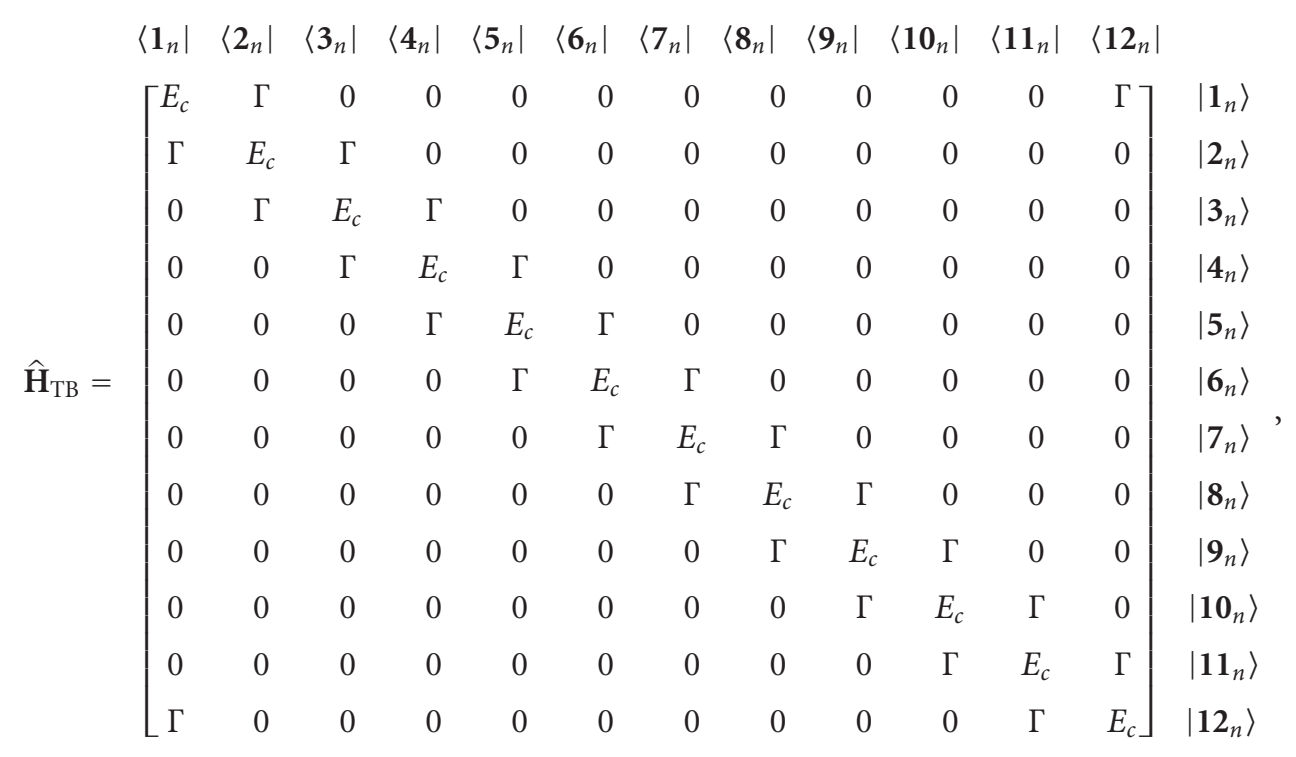

where state $\left|\mathbf{1}_{n}\right\rangle$ corresponds to the Wannier function localized in the first cavity forming the CCW, and state $\left|2_{n}\right\rangle$ corresponds to the Wannier function localized in the second cavity forming the waveguide, and so on. The diagonal matrix element for each site is $E_{c}$, this is the energy of the resonant mode for each cavity. Periodic boundary conditions are expressed at the waveguide ends as $\hat{\mathbf{a}}_{1}^{\dagger}=\hat{\mathbf{a}}_{12}^{\dagger}$ and $\hat{\mathbf{a}}_{1}=\hat{\mathbf{a}}_{12}$.

2.2. Dispersion, Group Velocity, and GVD. It is assumed that the wavelength of the single photon $\lambda_{\text {ph }}$ of $1.182 \mu \mathrm{m}$, which is a realistic wavelength for a GaAs/InGaAs-based QD emitter. This wavelength corresponds to the cavity resonant frequency $\omega_{c}=1.594 \cdot 10^{15} \mathrm{rad} / \mathrm{s}$, where $E_{c}=$ $\hbar \omega_{c}$. Next, assuming a quality factor $Q=1000$ for each cavity of the CCW, the coupling rate between neighboring cavities is thus calculated to be $\Gamma=1.594$. $10^{12} \mathrm{rad} / s$, where $\Gamma$ is defined as $\omega_{c} / Q$. This definition of $\Gamma$ assumes that coupling to the nearest neighbor cavity is the only loss channel, that is, out-of-plan losses are ignored.
The dispersion relation of this CCW is solved from the diagonalization of the Hamiltonian matrix. Although working in a real space representation, the problem is fully equivalent to the reciprocal space-based Bloch representation. In fact, for $N$ sites, $N k$ points exist in reciprocal space and are defined as $k=2 \pi \mathrm{K} / \mathrm{Na}$ where $\mathrm{K}$ is a quantum number such that $\mathbf{K}=-N / 2+1, \ldots, N / 2$ and $a$ the lattice constant of the periodic waveguide. As a result, the same energy eigenvalues are obtained. They are plotted in red for the normalized positive $k$ points of the first Brillouin zone in Figure 2.

The dispersion obtained numerically matches very well the theoretical dispersion for CCWs in (4) in the linear dispersion approximation. Under this approximation, we assume weak coupling, which means that photons may only leak into the nearest neighbor cavity [14-18]. The tightbinding model yields the optical carrier frequencies

$$
\omega_{k}=\omega_{c}[1+\kappa \cos (k a)],
$$

where $\omega_{c}$ is the resonant frequency of a single cavity, $\kappa$ is the coupling coefficient between cavities, $k$ is the Bloch wave 


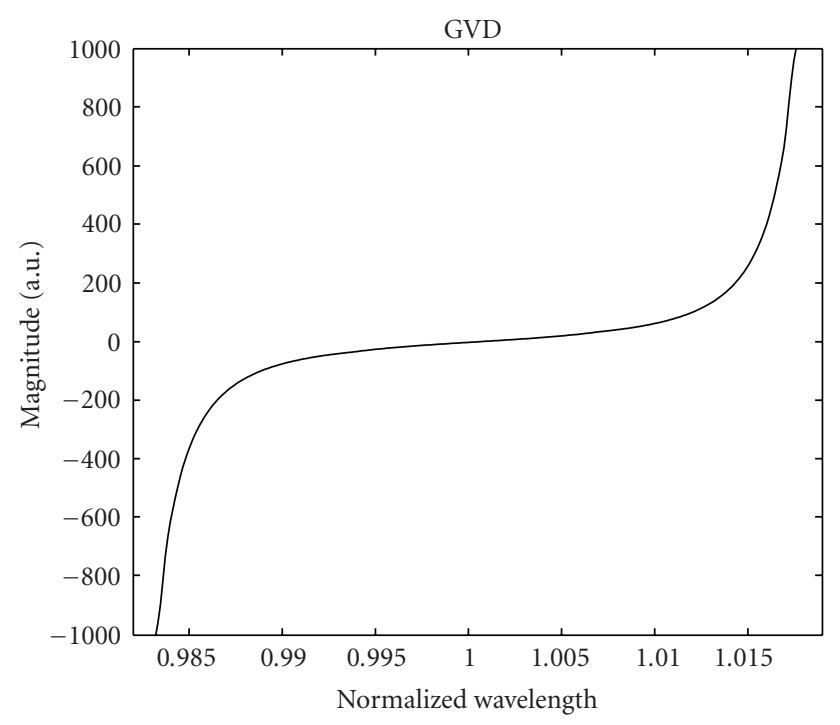

FIgURe 4: Group Velocity Dispersion.

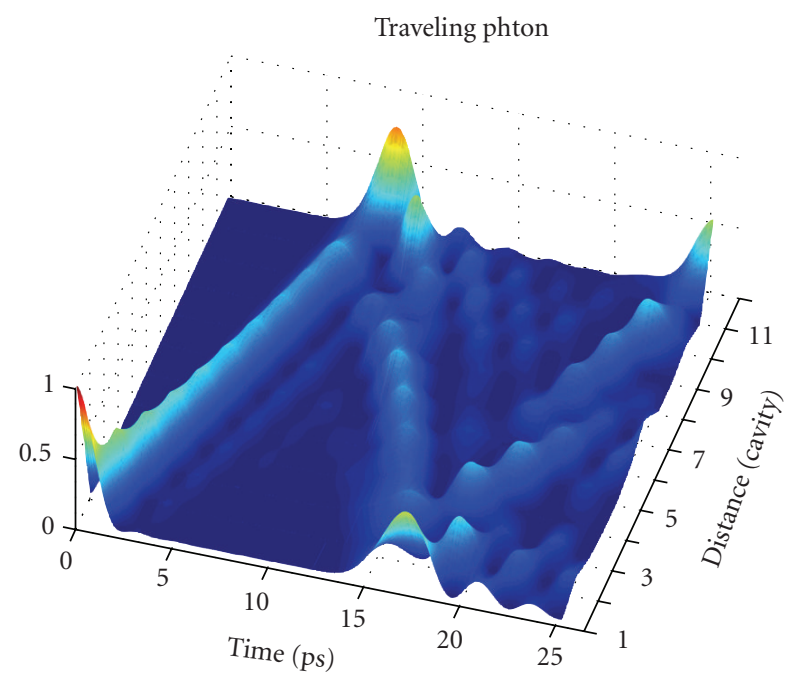

FIgUre 5: Traveling Photon.

number, and $a$ is the lattice constant. For this waveguide, the coupling coefficient was found to be $\kappa=-0.002$.

An expression for the group velocity $v_{g}$ can be derived from (4) as follows:

$$
v_{g}=\nabla_{k} \omega_{k}=-\omega_{c} \kappa a \sin (k a)
$$

The group velocity $v_{g}$ normalized over $c$ (the speed of light in vacuum) is plotted in Figure 3 throughout the normalized coupled cavity waveguide band. It is obtained by taking the derivative of obtained energy eigenvalues with respect to $k$. At the edges of the waveguide band, that which corresponds to when $k$ is 0 or 1 , the group velocity tends toward zero.

This result has two important consequences. First, the group velocity dispersion is ill defined when $k$ is 0 or 1 according to the standard definition in (6). Second, the

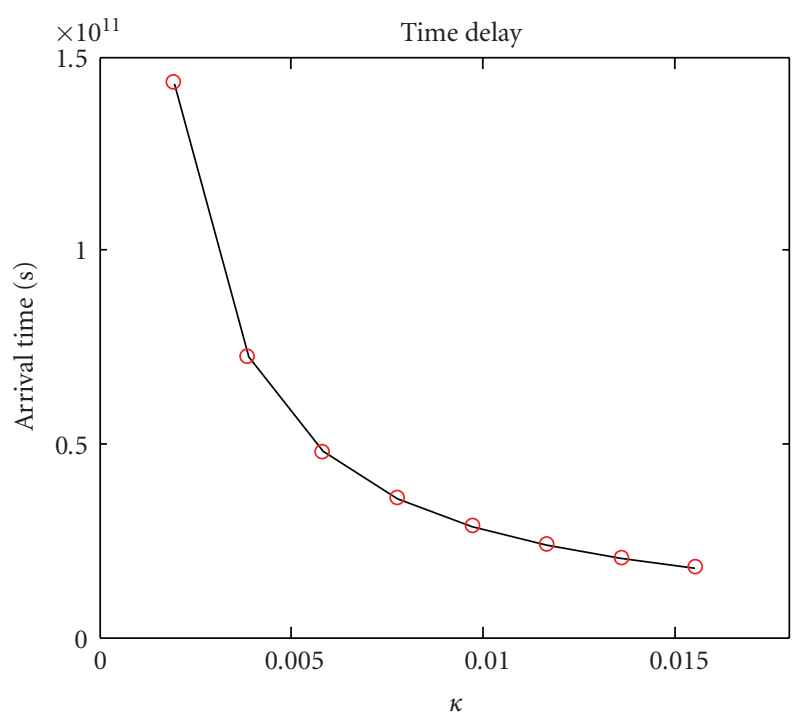

Figure 6: Time Delay.

group velocity dispersion may be either positive or negative depending on which band edge $k$ is closest to

$$
\mathrm{GVD}=-\left(\frac{2 \pi c}{\lambda^{2}}\right) \frac{d}{d \omega}\left(\frac{1}{v_{g}(k)}\right)
$$

The group velocity dispersion (GVD) for the 12 cavities forming the waveguide is plotted in Figure 4 within the CCW band. For practical applications, Mookherjea derived more appropriate definitions of GVD that satisfy a small fractional change of the GVD coefficient over the range of frequencies of interest [19].

2.3. Single-Photon Propagation. A single-photon pulse is shown propagating for the waveguide composed of $12 \mathrm{cav}-$ ities in Figure 5. The time dependence for the coupled-cavity waveguide is obtained numerically using the Louiville or Von Neumann Equation (similar to the approach discussed in [20]) in conjunction with the tight-binding Hamiltonian to solve for the time evolution of the density matrix whose states correspond to the Wannier functions localized in the cavities forming the waveguide. It is assumed that there are neither any out-of-plane losses or material absorption causing the CCW mode to decay nor any scattering resulting in a sudden change of the phase.

The single photon pulse can be seen traveling from cavity 1 to Cavity 12 in about 9 picoseconds before being reflected. An oscillatory structure at the trailing edge of the pulse can be noticed. We believe this feature has to do with the singlephoton pulse defined initially at a precise point in space and time. The consequence is a wideband single-photon pulse, and therefore higher-order terms can no longer be neglected resulting in envelope distortion. It is also helpful to compare that propagation time with the decoherence time of atoms or QDs. The time it takes to transfer a photon from a source cavity to a target cavity is a relatively fast process compared to the decoherence processes associated 


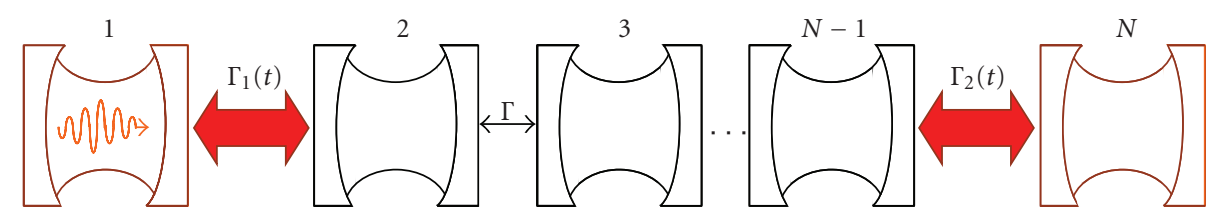

FIGURE 7: Unloading/loading by means of time-varying coupling coefficients.

with electron spin qubits in self-assembled QD (our storage qubit of interest). In a recent publication of ours [20], we showed how the coherent exchange between a photon qubit and an electron spin qubit in a QD is affected by decoherence resulting from hyperfine interactions. Although these decoherence processes prevented the interacting qubits from reaching maximum entanglement, there were at least partially entangled. It is important to note that these interactions were taking place over a longer period of time, about 1 to 2 orders of magnitude longer than the time needed for a photon to propagate down 12 cavities. In addition, a novel scheme based on ultrafast optical spin echo shows that decoherence times on the order of microseconds are achievable [21]. Therefore, it is anticipated that the propagation time of the photon will not be much of an issue over small to medium range distances.

Figure 5 also depicts the single-photon pulse propagation in the linear region of the CCW; therefore, the single-photon frequency is tuned to the middle of the waveguide band. (This corresponds to a normalized frequency equal to 1 in Figure 2.) As a result, ignoring the oscillatory structure at the trailing edge of the pulse, a pulse propagates mostly unchanged in shape. On the other hand, when the singlephoton pulse propagation is determined by the flat region of the CCW dispersion corresponding to one of the edges of the transmission band, there exists a considerable slowing of the group velocity. Figure 6 depicts the travel time from cavity 1 to cavity 12 as a function of coupling coefficient $\kappa$.

2.4. Engineering Cavity-Waveguide Couplings. Dynamically coupling the end cavities to the waveguide can be achieved in two different ways. On one hand, this can be done by setting all the resonant modes to be the same for all cavities including the end cavities while varying the coupling coefficients between the end cavities and the waveguide in time in order to load and unload the photon from and onto the waveguide. This is depicted in Figure 7, and its corresponding Hamiltonian is described in

$$
\begin{aligned}
\hat{\mathbf{H}}= & \sum_{j} \hbar \omega_{c} \hat{\mathbf{a}}_{j}^{\dagger} \hat{\mathbf{a}}_{j}+\sum_{2}^{N-2} \Gamma\left(\hat{\mathbf{a}}_{j}^{\dagger} \hat{\mathbf{a}}_{j+1}+\hat{\mathbf{a}}_{j+1}^{\dagger} \hat{\mathbf{a}}_{j}\right) \\
& +\Gamma_{1}(t)\left(\hat{\mathbf{a}}_{1}^{\dagger} \hat{\mathbf{a}}_{2}+\hat{\mathbf{a}}_{2}^{\dagger} \hat{\mathbf{a}}_{1}\right)+\Gamma_{2}(t)\left(\hat{\mathbf{a}}_{N-1}^{\dagger} \hat{\mathbf{a}}_{N}+\hat{\mathbf{a}}_{N}^{\dagger} \hat{\mathbf{a}}_{N-1}\right) .
\end{aligned}
$$

On the other hand, dynamic coupling can also be completed by setting coupling coefficients to be the same for all cavities including the end cavities while varying the resonant modes of cavity 2 and cavity $N-1$ in time in order to load and unload the photon from and onto the waveguide. This is depicted in Figure 8, and its corresponding Hamiltonian is described in

$$
\begin{aligned}
\hat{\mathbf{H}}= & \sum_{j \neq 2, N-1} \hbar \omega_{c} \hat{\mathbf{a}}_{j}^{\dagger} \hat{\mathbf{a}}_{j}+\hbar \omega_{2}(t) \hat{\mathbf{a}}_{2}^{\dagger} \widehat{\mathbf{a}}_{2}+\hbar \omega_{N-1}(t) \hat{\mathbf{a}}_{N-1}^{\dagger} \hat{\mathbf{a}}_{N-1} \\
& +\sum_{1}^{N-1} \Gamma\left(\hat{\mathbf{a}}_{j}^{\dagger} \hat{\mathbf{a}}_{j+1}+\hat{\mathbf{a}}_{j+1}^{\dagger} \hat{\mathbf{a}}_{j}\right) .
\end{aligned}
$$

In practice, both of these approaches can be realized by means of a spatial modulation of the refractive index within the quantum network. In order for these loading and unloading operations to be feasible in a functioning quantum network though, they must be performed at speeds much greater than usual decoherence possesses therefore requiring the assistance of ultrafast optical pulses. Methods for the dynamical tuning of refractive index are based on nonlinear effects, carrier injection by linear absorption of an optical pump (free-carrier plasma dispersion effect), carrier injection using a PIN diode, or thermal tuning via optical heating. We are interested in carrier injection by linear absorption of an optical pump which many groups have shown to be a viable method. For example, Tanaka et al. demonstrated a change in cavity $Q$ from 12,000 to 3,000 in 4 ps [22]. Lipson's group at Cornell University used a $100 \mathrm{fs}$ pump pulse to generate a $18 \mathrm{ps}$ index change pulse [23] and also demonstrated how a 1.5 ps pump pulse corresponding to a $25 \mathrm{ps}$ index change pulse could change the Q factor of a cavity from 60000 to 17000 [24]. Tanabe et al. generated a 14 ps pump pulse resulting in a change in photon lifetime from $320 \mathrm{ps}$ to $70 \mathrm{ps}$ inside a high $Q$ cavity [25]. Carrier injection using PIN diode is an interesting alternative and allows for both the injection and extraction of carrier simultaneously. The resulting shape of the free carrier index change looks a lot like a square pulse. Gardes et al. showed a $7 \mathrm{ps}$ rise and fall time in the index change was possible [26].

2.5. Transfer Efficiencies. On-chip on-demand single-photon transfer stipulates that the photon is unloaded onto a waveguide from a cavity 1 at a time $t_{1}$ allowing it to travel down the waveguide before being loaded into a cavity 2 at a time $t_{2}$. Considering the two approaches mentioned in the previous section, a critical question to be answered is what transfer efficiencies can be obtained.

First, we consider the case of time-varying coupling coefficients. The unloading of the photon onto the waveguide is achieved by dynamically switching the magnitude of the coupling coefficient between cavity 1 and cavity 2 starting at $t_{1}$. Perhaps, this can be realized using an approach similar to Noda's [22]. Then, the loading of the photon from 


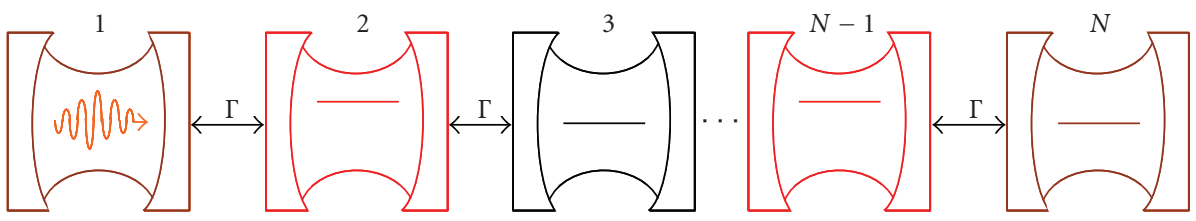

FIGURE 8: Unloading/loading by means of time-varying resonant frequencies

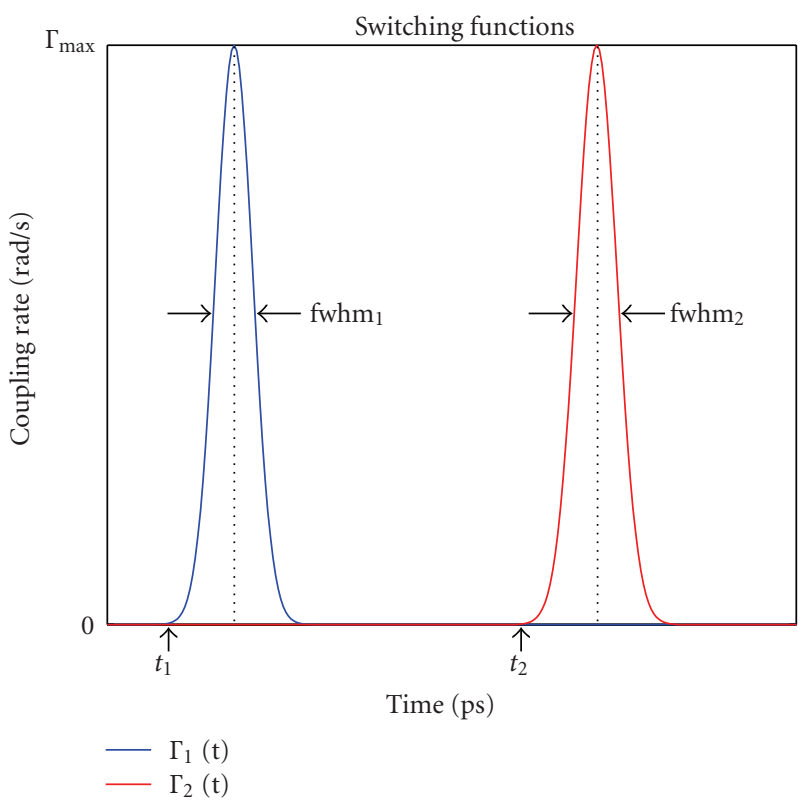

FIGURE 9: Switching functions $\Gamma_{1}(t)$ and $\Gamma_{2}(t)$.

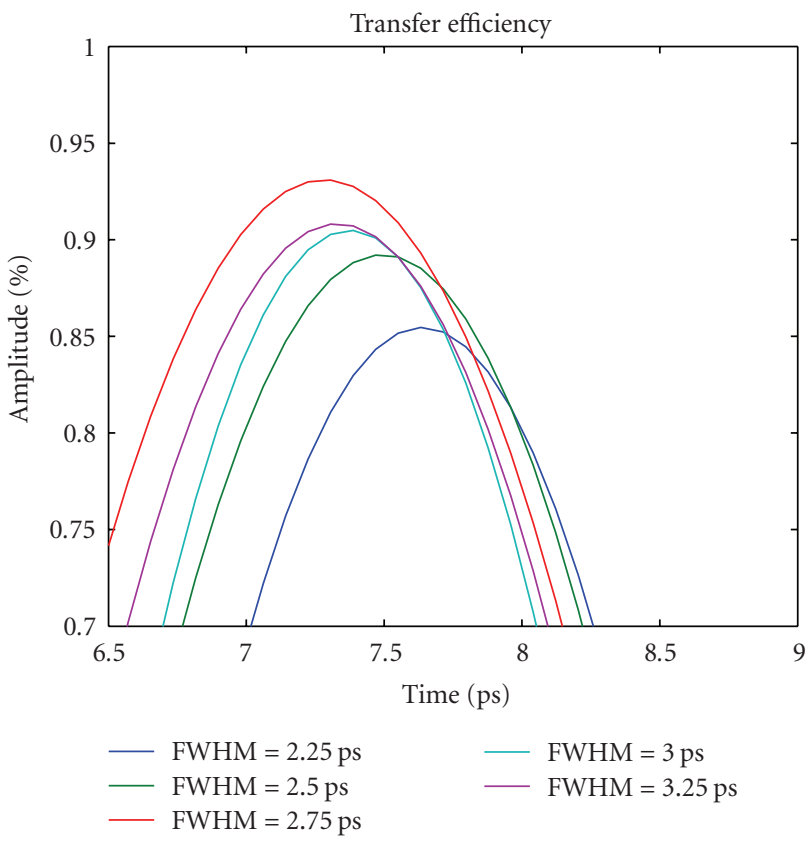

FIGURE 10: Transfer efficiency with time-varying coupling coefficients.

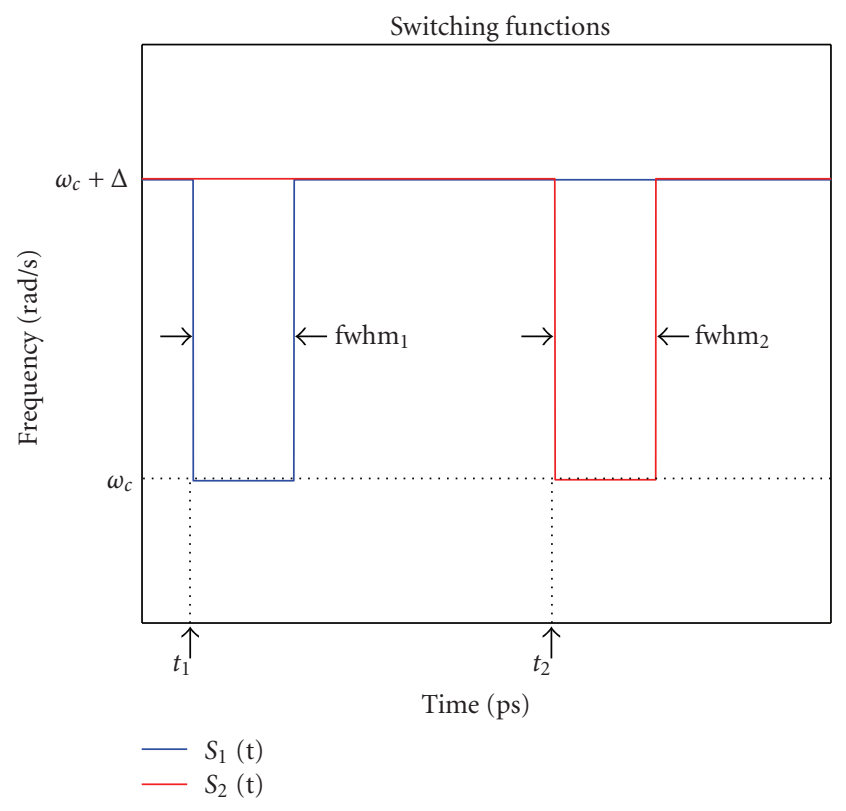

FIGURE 11: Switching functions $S_{1}(t)$ and $S_{2}(t)$.

the waveguide into cavity $N$ is achieved by once again dynamically switching the magnitude of coupling coefficient between cavity $N-1$ and cavity $N$ starting at $t_{2}$.

Design parameters of interest for both switching functions, $\Gamma_{1}(t)$ and $\Gamma_{2}(t)$, are their shape or time dependence, the range of coupling strength over which they are varied, the window in time over which they should be varied. There are also few design constraints. The temporal width of $\Gamma_{1}(t)$ or FWHM should be larger than the natural cavity decay into the CCW so as to allow the photon to escape yet smaller than round trip time. Similarly, properties of $\Gamma_{2}(t)$ will greatly depend on factors such as the CCW length, the group velocity, or the group velocity dispersion. Figure 9 shows the switching functions $\Gamma_{1}(t)$ and $\Gamma_{2}(t)$ qualitatively.

It was found that the optimum shape of switching functions $\Gamma_{1}(t)$ and $\Gamma_{2}(t)$ is a Gaussian profile. In a CCW with constant coupling between its cavities, when a photon is allowed to propagate freely, though highly localized initially (effectively represented by a delta function in space), it eventually exhibits a distribution in space that happens to be well approximated by a Gaussian. It may be that the distribution could be also approximated by other functions, for example, a squared hyperbolic secant. The authors believe it is worth further investigating the mechanism behind the broadening associated with the probability of finding the 


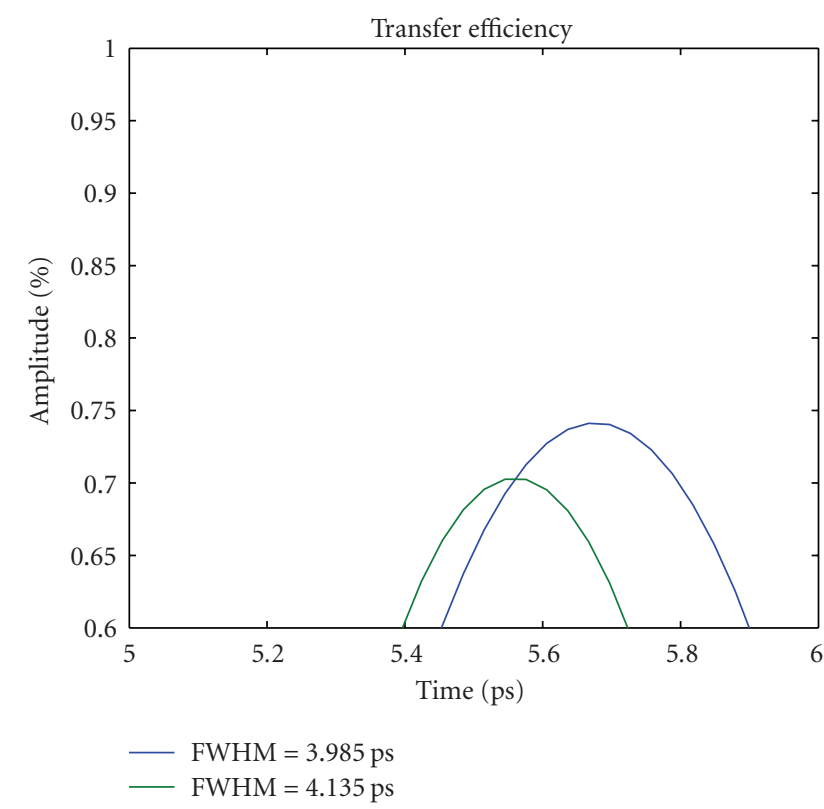

FIGURE 12: Transfer efficiency with time-varying resonant frequencies.

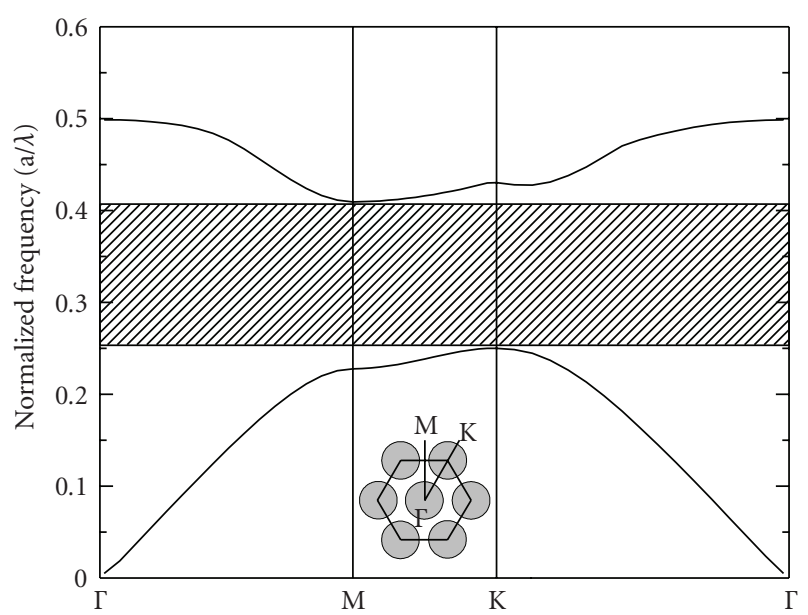

FIGURE 13: TE band diagram of a 2D photonic crystal with circular scatterers in a triangular lattice. The structure simulated here is a hole type crystal of index 3.4.

photon in space. In any case, this is certainly related to the fact that the coupled cavities have a finite $Q$ which introduces an uncertainty in the time over which the photons actually hops from one cavity to the next. That being said, the context of Figure 9 is slightly different as it relates not just to a waveguide with constant coupling coefficient but to time-varying coupling coefficients. In other words, the coupling constant between two quasi-bound modes that are degenerate in frequency is varied. And the cavity $Q$ depends on the coupling $\Gamma(t)$ where $Q=\omega / \Gamma(t)$. Providing that the switching function $\Gamma(t)$ has a Gaussian shape, the $Q(t)$ will also have a Gaussian shape, thus allowing for minimum reflections at the target cavity boundaries since the incoming

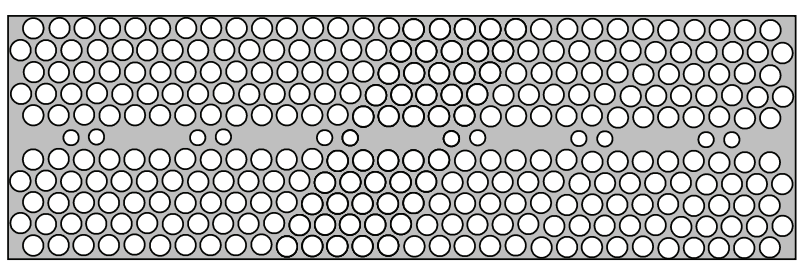

FIGURE 14: Structure of the high- $Q$ cavity intended as a classical embodiment to the $j=1$ and $j=N$ cavities in the quantum model above.

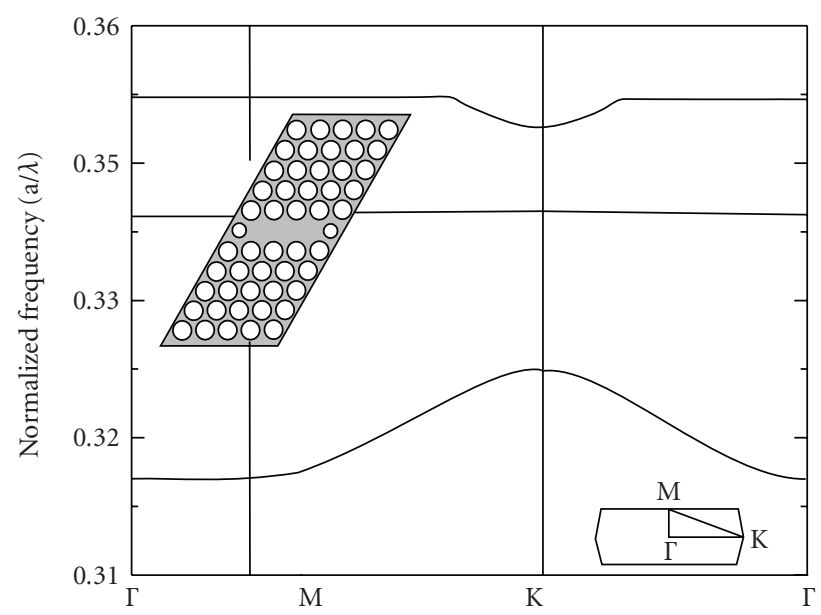

FIGURE 15: PWE calculation of our defect mode of interest over the full first Brillouin Zone. The cavity supercell is shown in the inset. There are only confined, nondispersive modes in the M crystal direction but along the $\mathrm{K}$ crystal direction, our intended direction of CCW propagation, dispersion is observed.

photon also has a Gaussian-like probability distribution in time.

As far as the range over which the coupling strength should be varied for $\Gamma_{1}(t)$, it is assumed that there is not any coupling, initially, between cavity 1 and cavity 2 , yielding $\Gamma_{1}(0)=0$. However, the maximum of the Gaussian shaped switching function is designed to be $\Gamma_{\max }=1.594$. $10^{12} \mathrm{rad} / \mathrm{s}$, which corresponds to the regular coupling rate between neighboring cavities of the CCW. The minimum FWHM for $\Gamma_{1}(t)$ that allows the entire photon to leak out of the cavity was found to be 3 ps. The characteristics of $\Gamma_{2}(t)$ are engineered so as to maximize the transfer efficiency.

Figure 10 shows transfer efficiencies for various FWHM for $\Gamma_{2}(t)$ and a large range of $t_{2}$. These transfer efficiencies are calculated assuming $\Gamma_{1}(t)$ with a FWHM equal to $3 \mathrm{ps}$, a peak coupling rate equal to $1.594 \cdot 10^{12} \mathrm{rad} / \mathrm{s}$, and $t_{1}$ equal to $1.5 \mathrm{ps}$. A maximum transfer efficiency of $93 \%$ is obtained for $\Gamma_{2}(t)$ with a FWHM equal to $2.75 \mathrm{ps}$ and a starting time $t_{2}$ equal to $7.25 \mathrm{ps}$. The transfer efficiency is defined as the ratio of the probability of finding a photon in cavity $N$ following its capture (once $\Gamma_{2}(t)$ goes back to zero) over the probability of finding a photon in Cavity 1 before its release (when $\Gamma_{1}(0)=$ 0 ), which is always unity.

Next, the case of time-varying resonant frequencies is considered. The unloading of the photon onto the waveguide 


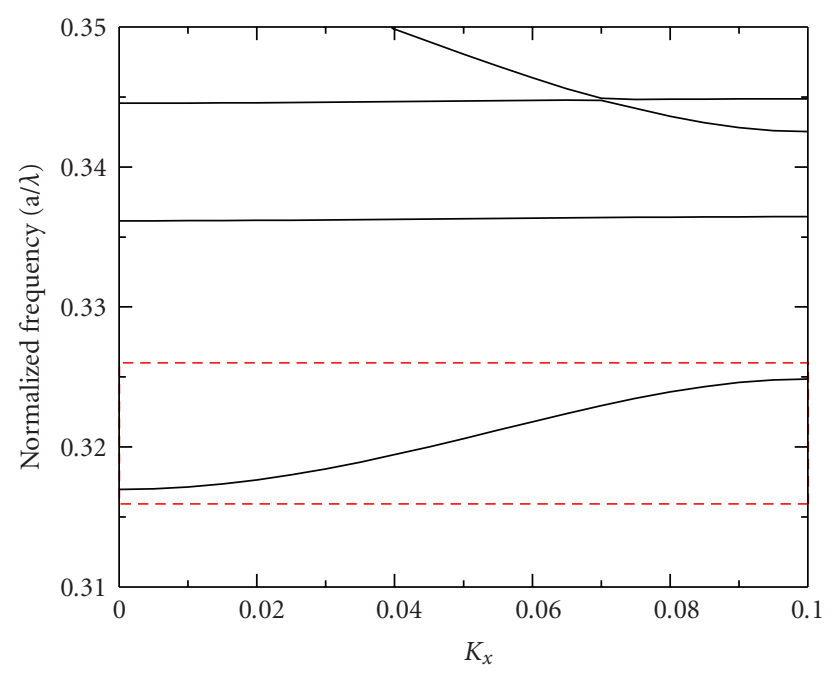

(a)

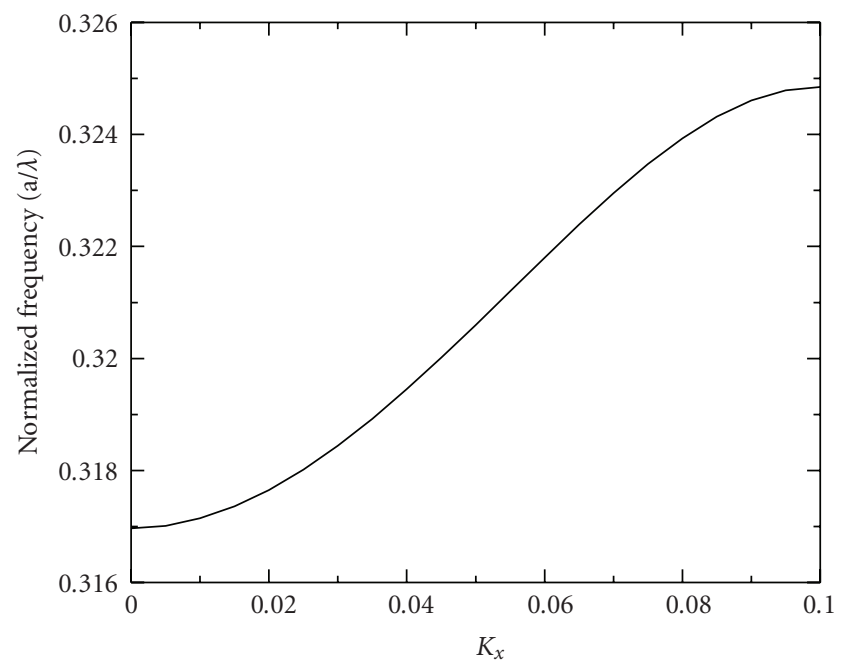

(b)

Figure 16: Dispersion relation for the L3s2 CCW structure described above. (a) we see that many modes exist in the crystal's photonic band gap, some with high confinement that result in no dispersion and others that allow coupled-cavity resonance for propagation that result in the sinusoidal k-relation from (4). The mode of interest is selected and zoomed in upon for (b).

is achieved by dynamically switching the resonant frequency of cavity 2 from $\omega_{c}+\Delta$ to $\omega_{c}$ starting at $t_{1}$. Then, the loading of the photon from the waveguide into cavity $N$ is achieved by dynamically switching the resonant frequency of cavity $N-1$ from $\omega_{c}+\Delta$ to $\omega_{c}$ starting at $t_{2}$ and subsequently from $\omega_{c}$ back to $\omega_{c}+\Delta$.

Design parameters of interest for both switching functions, $S_{1}(t)$ and $S_{2}(t)$, are their shape or time dependence, the range of frequencies over which the cavity resonant frequencies are varied, the time window over which resonant frequencies should be varied. As far as design constraints, it is desirable that the amount of detuning $\Delta$ for the resonant frequency of the "barrier" cavities to be larger than the waveguide bandwidth to avoid any significant

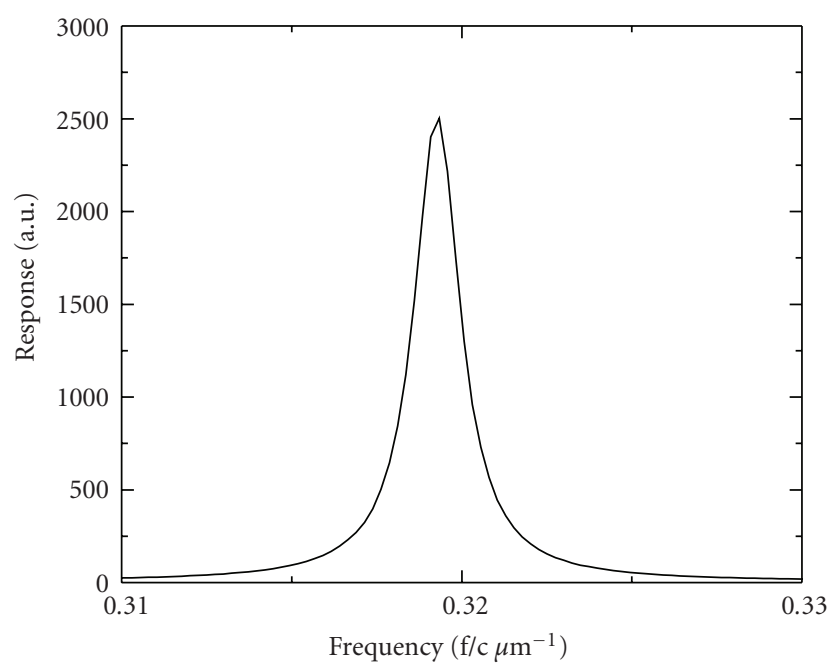

(a)

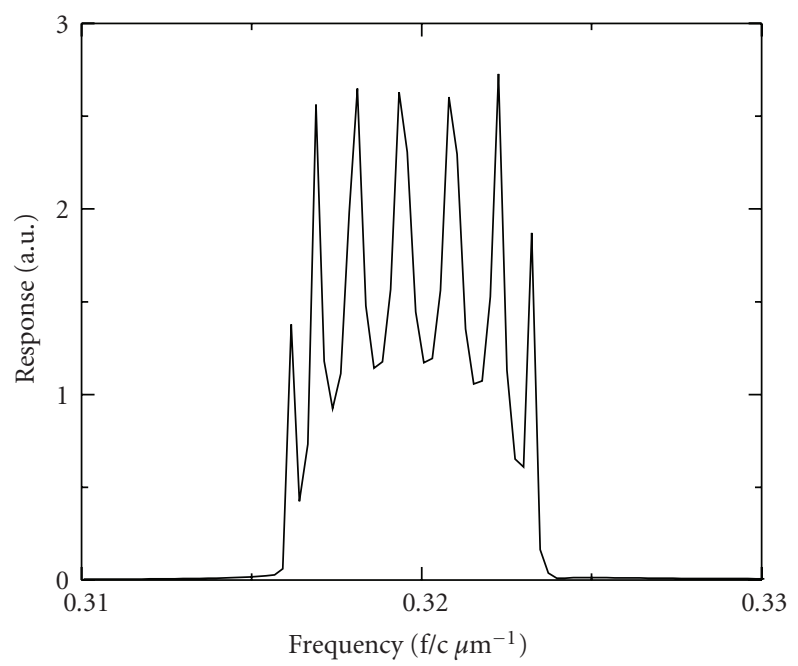

(b)

FIGURE 17: (a) represents the spectra inside a single L3s cavity isolated from any other cavities, while (b) represents the spectra through a 7 cavity coupled L3s2 system. Results calculated from FDTD.

coupling between the waveguide and the end cavities. Also, the duration of $S_{1}(t)$, which is the time during which the resonant frequency of cavity 2 is switched from $\omega_{c}+\Delta$ to $\omega_{c}$, should be large enough for the photon to escape, yet smaller than the round trip time. Similarly, $S_{2}(t)$ needs to be large enough for the photon to be captured. Figure 11 shows $S_{1}(t)$ and $S_{2}(t)$ qualitatively.

It was found that the optimum shape for $S_{1}(t)$ and $S_{2}(t)$ is a square profile. In the case of the time-varying frequency scheme, the coupling is mostly dictated by $\omega(\Gamma \ll \omega)$, so until the frequency of the "barrier" cavity matches the frequencies of adjacent cavities, the probability of tunneling through is insignificant. That explains why a square profile is more appropriate. Earlier, in the case of the time-varying coupling coefficient scheme, the difference was that all the $\omega$ 's were the same; therefore, the probability of tunneling 

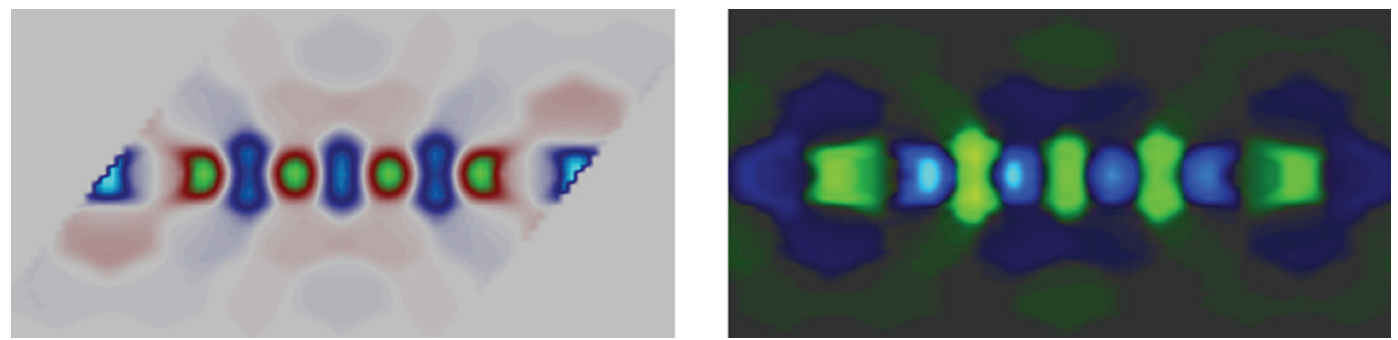

FIGURE 18: Simulated CCW modes in the L3s2 system. On the left, a PWE calculation of the allowed mode corresponding to the dispersive mode in Figure 15 matches well with the FDTD simulation of the propagating mode on the right, both showing the out of plane field component (Hy).

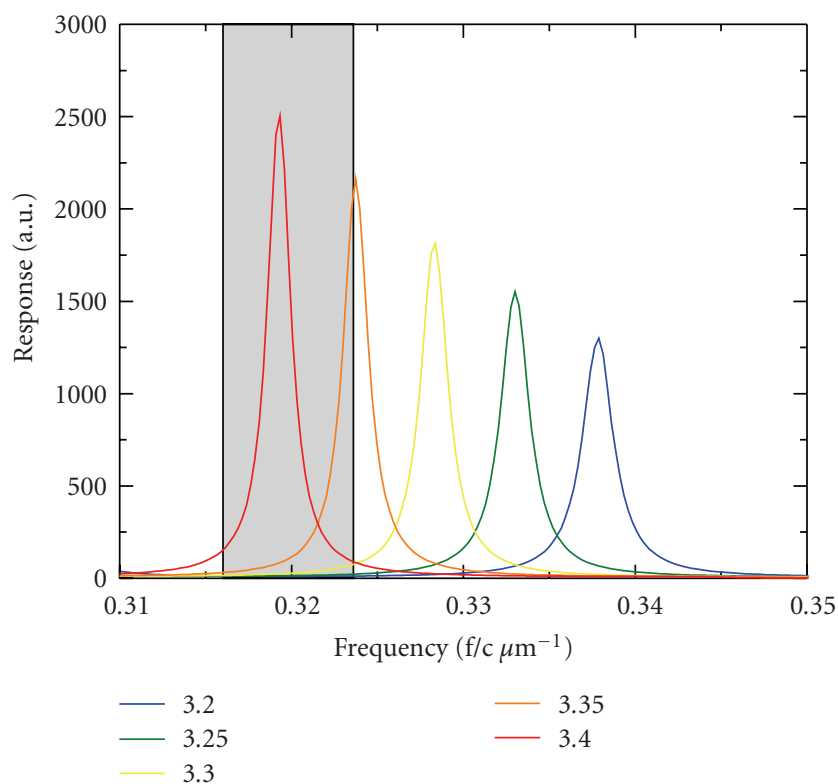

Figure 19: Cavity resonance shifting due to a change in material index of refraction. Using a standard index of 3.4 (red) as our base, to comply with $\mathrm{Si}$ or GaAs substrates, switching the barrier regions of our heterostructured cavity to an index of 3.3 (yellow) offers negligible overlap between "barrier" cavity resonances and the waveguide bandwidth (grey). Further confinement would be possible with lower switched index but is less physically reasonable.

through was only depending on $\Gamma(t)$. In fact, much higher transfer efficiencies were achieved for the time-varying frequency scheme with a square shape switching function with the prescribed duration (about 75\%) compared to a Gaussian shape switching function with a wide range of duration (no more than 10\%). The authors believe that the transfer efficiency could further be improved if the switching function was switched on and off adiabatically, resulting in a rounded square shape. In addition, the switching function duration is now chosen to correspond to approximately twice the photon lifetime in the "barrier" cavity since the photon has to both enter and exit the "barrier" cavity before it can reach the target cavity.

As far as the range over which the resonant frequency of the "barrier" cavities should be varied, we assume that each cavity in the CCW has a $Q$ of 1000 resulting in a coupling coefficient of $\Gamma=1.594 \cdot 10^{12} \mathrm{rad} / \mathrm{s}$ at the wavelength of interest and a bandwidth of $\mathrm{BW}=\omega \pm \kappa=1 \pm 0.002$ in normalized units of frequency. In our case, this corresponds to $\mathrm{BW}=1.59 \cdot 10^{15} \pm 3.18 \cdot 10^{12} \mathrm{rad} / \mathrm{s}$. Consequently, we designed the detuning parameter to be $\Delta=3.24 \cdot 10^{12} \mathrm{rad} / \mathrm{s}$ such that $|\kappa| \leq \Delta \ll \omega$. Therefore, by switching "barrier" cavity frequencies from $\omega_{c}$ to $\omega_{c}+\Delta$, we are able to prevent coupling between the end cavities and the CCW.

Figure 12 shows transfer efficiencies for various FWHM for $S_{2}(t)$ and a large range of $t_{2}$. These transfer efficiencies are calculated assuming $S_{1}(t)$ with a FWHM equal to $4.875 \mathrm{ps}$ and $t_{1}$ equal to $1.5 \mathrm{ps}$. A maximum transfer efficiency of $75 \%$ is obtained for $S_{2}(t)$ with a FWHM equal to 3.985 ps and a starting time $t_{2}$ equal to $5.7 \mathrm{ps}$.

\section{Physical Design}

As a sort of proof of principal, a classical optical system was designed to compare and contrast the propagation properties of light in CCW systems. Using photonic crystal as a means to integrate such a coupled system allows great potential for high density integration of waveguides and high- $Q$ cavities that are readily reproducible in fabrication. There has been much work showing from both theory and experiment, that photonic crystal CCW systems can exploit the unique dispersive properties discussed previously for applications of slow light pulse compression and transparency [27-30]. Many different systems have been shown to stop light including traditional defect waveguides with side-coupled integrated sequence of resonators. Initially, these systems seem identical to CCW structures; they both exhibit a cosine-like dispersion relation inside the photonic bandgap, which is eventually flattened adiabatically to stop the light. Differences become apparent when one consider the photonic wave function. In the CCW system under consideration, a highly localized photonic wave function is anticipated once the light is stopped, that is, within a single cavity. On the other hand, for defect waveguides with side-coupled integrated sequence of resonators, the photonic wave function would be spread over the defect waveguide and several cavities. For the reasons just mentioned, a CCW structure decrease device footprint. In addition, CCW structures lend themselves more readily to cavity QED applications. In this publication we focus on the maneuvering of light through a network of cavities only. 


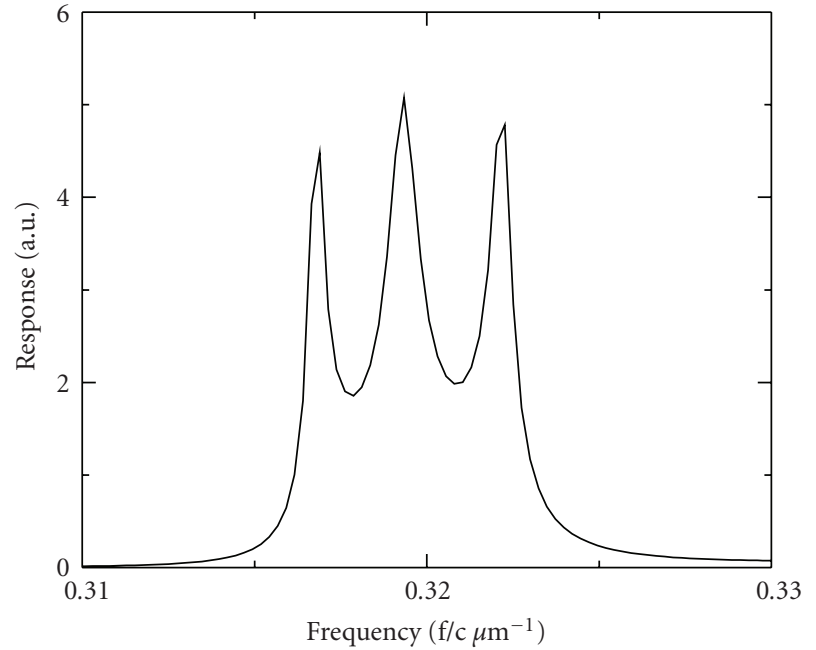

(a)

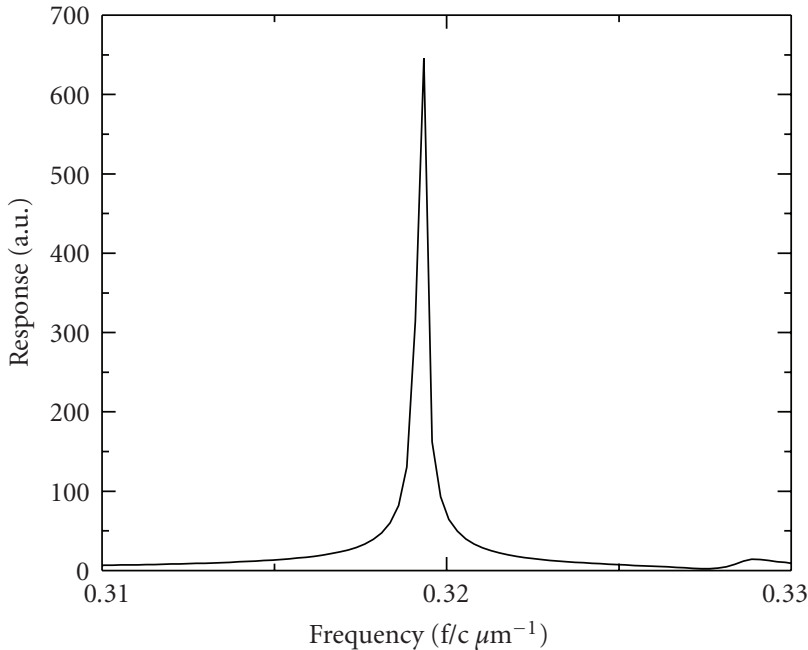

(b)

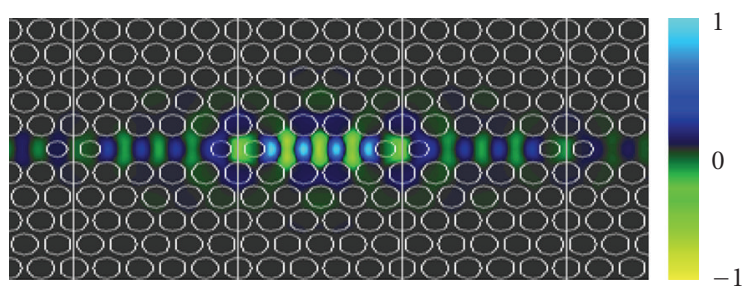

(c)

FIgURE 20: (a) spectra of 3 coupled cavities simulated through FDTD. (b) the first and third cavities have been index switched to show spectra that now represents an isolated heterostructured High- $Q$ cavity. The spatial mode profile of this High- $Q$ mode is also shown in (c).

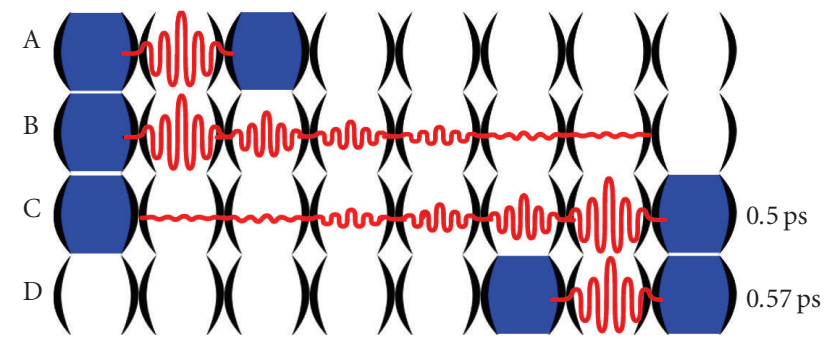

FIGURE 21: High $Q$ cavity mode release and capture process. A shows the confined cavity with both barriers activated with an index of 3.3. B shows the opening of the inside cavity by index switching to $n=3.4$. With the second cavity's outside barrier closed, C, the energy accumulates and is then trapped by closing the inner barrier of the second cavity in D.

The coupling of cavity modes to waveguide modes has been a topic of intense research effort for the realization of both quantum and classical integrated optical circuit $[11,31,32]$. It is therefore useful, in photonic crystal devices, to design similarities between isolated cavity modes and propagating CCW modes. This similarity makes for efficient coupling toward the effort of release and capture of optical energy as well as bends and splitters for waveguide routing $[33,34]$. The bandwidth of a CCW band may also be adjusted by changing localization properties of the cavities, or the coupling strength (overlap integral) between the localized cavity modes. For instance, decreasing the intercavity distance leads to a wider bandwidth [35].

With many such degrees of design freedom that impact the modes of both the isolated cavity and the CCW, use of photonic crystal cavities for analysis of a coupled cavity system is a fruitful choice. The remainder of this section will review the methods and findings of the design and characterization of a CCW system with isolated cavity release and capture switching ability.

3.1. Numerical Methods. Analysis of our classical system makes use of the standard numerical simulation tools employed in photonic crystal devices, Plane Wave Expansion (PWE), and Finite-Difference Time Domain (FDTD). The proposed embodiment of this CCW system in a photonic crystal featuring a triangular lattice of circular holes will be performed for TE (even) polarized light only. This choice of polarization allows both a more comfortable band gap in which to engineer defects as well as a more accurate approximation from a $3 \mathrm{D}$ slab to a $2 \mathrm{D}$ effective index method approximation for future work. All results presented herein may be taken as approximations to a 3D finite slab or simply as a proof of concept in an infinite 2D system. The potential barriers and loss mechanisms for moving to a fully $3 \mathrm{D}$ model will be discussed later.

PWE provides an initial glimpse of the resonant behavior of these index-periodic metamaterials. The band in which the structure exhibits this periodicity-induced resonance, as 


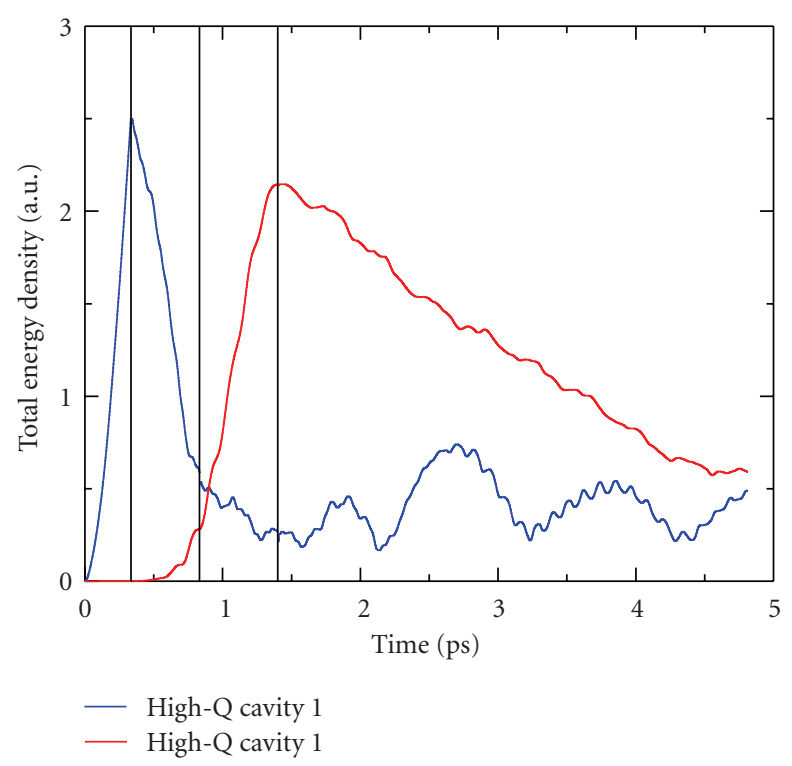

(a)

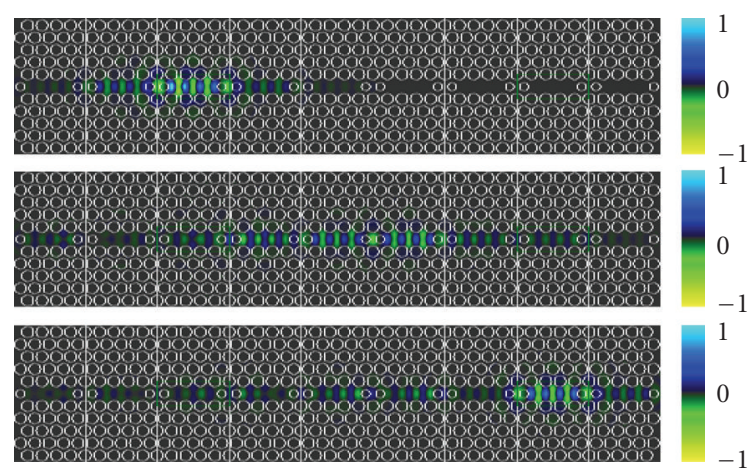

(b)

FIgURe 22: Three step release of an optical mode in a high- $Q$ cavity into a CCW and capture into a second high-Q cavity with $77 \%$ efficiency. Energy density (a) and field evolution (b) are shown to illustrate the process.

shown in Figure 13 represents a range of frequencies in which light is not allowed to propagate in the structure.

It is then, in this forbidden spectral range that engineered crystal defects lead to allowed optical modes that may be localized (cavities) or allowed to propagate (waveguides). PWE will also provide the means to find the dispersion relation for our coupled-cavity waveguide modes which is key to the calculation of GVD.

Finally, FDTD is implemented to study the spectral response as well as the operational efficiency of these devices. This simulation tool allows the designers to observe how their device would operate under perfect conditions.

3.2. Structure. The envisioned system will consist of coupled cavities which exhibit guided modes as well as well-confined cavities that will exhibit high $Q$-factors in comparison. Lengths of CCW cavities will act as transmission lanes between the high- $Q$ cavities and so the resonances of the two structures will have to align. We choose, as a simple proof of concept, to implement L3s cavities for both the CCW unit cavity as well as the high $Q$ cavity. L3 is the representation of a defect line cavity of three missing holes, while the $s$ denotes that the holes on the ends of the cavity are reduced in size. This size reduction enables the designer to sculpt the cavity resonance but is not considered variable for the current study. The CCW will be a chain of these cavities as defects in a triangular array of air hole circles of $r=0.4 a$ with 2 hole spacers $(r=0.3 a)$ between each cavity as shown in Figure 14. The variable $a$ is the lattice spacing of holes in the crystal.

In order to create the optical isolation necessary between the high- $Q$ cavity and the CCW chain, barriers of variable index material will be used. These regions represent targets for optically induced refractive index switching using off resonant excitation [22].

3.3. Coupled Cavity Waveguide. Implementation of our PWE scheme with the above device shows that light will be nondispersive and therefore strongly confined along the $M$ crystal direction but will exhibit dispersion and therefore propagate along the $\mathrm{K}$ crystal direction, as shown in Figure 15.

By orthogonalizing the $k$-vectors calculated in the PWE scheme so as to consider only the $\mathrm{K}$ direction, we may increment the PWE's eigenvalue calculations along only the direction of propagation to simulate the CCW structure's dispersion relation, shown in Figure 16.

The resulting spectral signature of this mode dispersion is shown in Figure 17 and will result in as many peaks over the range of resonant frequencies as there are coupled cavities in the chain.

Finally, it is important for the spatial field mode profiles of our cavity and CCW to be similar in order to maximize coupling between the two structures. Using both PWE and FDTD simulation methods, we verify the spatial field dependence shown in Figure 18 of our mode of choice.

3.4. Cavity. Relying on FDTD and the analysis of spectra, the design of our cavity is made through observation of the behavior of cavity resonance with changes to surrounding regions. First, an isolated L3s cavity is simulated to establish a basis for the types of modes this sort of cavity is likely to support. This sort of isolated defect is unable to be readily released into a CCW chain, and so a heterostructured cavity is implemented by including index-switchable regions to act as barriers to confine light away from the CCW portion of the device. Because the resonances of identical cavities shift in materials of different refractive index, shown in Figure 19, it is possible to use this resonance mismatch as a confining mechanism.

By implementing switched index cavities as barriers for the generation of a high- $Q$ cavity, the mode of interest for both barrier-open (left) and barrier-closed (right) three cavity segments are shown in Figure 20. While the heterostructured cavity is greatly diminished in $Q$-factor when compared to a single well isolated L3 cavity, it still represents a functional and well-confined optical mode that 
is effectively isolated from the neighboring CCW chain. With a Q-factor just under 2000, this proof of concept cavity represents an area for future research in optimization if strong coupling is desired.

Now, as we look toward coupling this cavity mode to the CCW chain, it should be noted that the cavity resonance of our isolated cavity is now centered to the CCW spectral feature. With these tools, the release and capture of an optical cavity mode is now outlined.

3.5. Implementing Coupling Mechanism. To perform release of the cavity mode into the CCW chain, one cavity barrier is index switched (opened) by optically induced carrier injection. Now matching the chain region, the cavity mode is allowed to couple to the CCW resonance and propagate from cavity to cavity down the chain. As the energy propagates down the chain, it becomes distributed amongst the coupled cavities and must be collected in a two step process by which the propagating mode is stopped by the outside secondary cavity barrier and allowed to accumulate before the inside secondary cavity barrier is activated, trapping the mode in the secondary heterostructured cavity. The full three step release and capture process is diagrammed here in Figure 21.

\section{Results and Discussion}

Through the switching protocol described above, transfer of energy from the optical mode in one high- $Q$ cavity, through a chain of 5 coupled cavities, to a second high- $Q$ cavity was achieved in a 2D FDTD scheme with $77 \%$ efficiency. Confinement at the first cavity, propagation between cavities, and capture in the second cavity is shown in Figure 22.

\section{Conclusion}

We have demonstrated that in theory using both a quantum model and a classical model that single photons can be transferred efficiently on-chip from a high- $Q$ cavity to another using coupled cavity waveguides if the dynamic coupling between nearest neighbor cavities is carefully engineered. From the quantum model, as far as singlephoton trapping and releasing mechanisms are concerned, dynamically switching coupling coefficients between "end" cavities and the waveguide yields much higher single photon transfer efficiencies (93\% versus $75 \%$ ) than dynamically switching the resonant frequencies of the "barrier" cavities. However, so far, only the single-photon trapping and releasing mechanism based on dynamically switching the resonant frequencies of the "barrier" cavities was able to be implemented in a practical way within our classical model. The endeavor to realize this system in a realistic form of a 3D photonic crystal slab (PCS) presents two hurdles: increased numerical cost, and out of plane loss mechanisms. It is expected that the increased numerical cost may be greatly alleviated by using 2D approximation methods for TE (even) polarization. However, due to the low group velocity of CCW modes and the zero group velocity of the high$Q$ state, careful engineering of the Fourier components of the field distributions in this system will be necessary to avoid coupling to out-of-plane radiative modes. Until then, though, the two models shown in this work yield strikingly similar single-photon transfer efficiencies $(\sim 75 \%)$, which reinforces the versatility of CCWs and the advantage of using such structures to implement photonic quantum networks.

\section{Acknowledgment}

The authors acknowledge support from NSF ECCS0725514 .

\section{References}

[1] B. B. Blinov, D. L. Moehring, L. M. Duan, and C. Monroe, "Observation of entanglement between a single trapped atom and a single photon," Nature, vol. 428, no. 6979, pp. 153-157, 2004.

[2] W. Yao, R. B. Liu, and L. J. Sham, "Theory of control of the spin-photon interface for quantum networks," Physical Review Letters, vol. 95, no. 3, Article ID 030504, 4 pages, 2005.

[3] W. Yao, R. B. Liu, and L. J. Sham, "Theory of control of the dynamics of the interface between stationary and flying qubits," Journal of Optics B, vol. 7, no. 10, pp. S318-S325, 2005.

[4] D. L. Moehring, M. J. Madsen, K. C. Younge et al., "Quantum networking with photons and trapped atoms (invited)," Journal of the Optical Society of America B, vol. 24, no. 2, pp. 300-315, 2007.

[5] T. Wilk, S. C. Webster, A. Kuhn, and G. Rempe, "Single-atom single-photon quantum interface," Science, vol. 317 , no. 5837, pp. 488-490, 2007.

[6] M. N. Leuenberger, M. E. Flatté, and D. D. Awschalom, "Teleportation of electronic many-qubit states encoded in the electron spin of quantum dots via single photons," Physical Review Letters, vol. 94, no. 10, Article ID 107401, 2005.

[7] A. A. Houck, D. I. Schuster, J. M. Gambetta et al., "Generating single microwave photons in a circuit," Nature, vol. 449, no. 7160, pp. 328-331, 2007.

[8] J. Majer, J. M. Chow, J. M. Gambetta et al., "Coupling superconducting qubits via a cavity bus," Nature, vol. 449, no. 7161, pp. 443-447, 2007.

[9] D. I. Schuster, A. A. Houck, J. A. Schreier et al., "Resolving photon number states in a superconducting circuit," Nature, vol. 445, no. 7127, pp. 515-518, 2007.

[10] M. A. Sillanpää, J. I. Park, and R. W. Simmonds, "Coherent quantum state storage and transfer between two phase qubits via a resonant cavity," Nature, vol. 449, no. 7161, pp. 438-442, 2007.

[11] D. England, A. Faraon, B. Zhang, Y. Yamamoto, and J. Vuckovic, "Generation and transfer of single photons on a photonic crystal chip," Optics Express, vol. 15, no. 9, pp. 5550$5558,2007$.

[12] J. Q. Liao, Z. R. Gong, L. Zhou, YU. X. Liu, C. P. Sun, and F. Nori, "Controlling the transport of single photons by tuning the frequency of either one or two cavities in an array of coupled cavities," Physical Review A, vol. 81, no. 4, Article ID 042304, 2010.

[13] P. Chak, J. E. Sipe, and S. Pereira, "Hamiltonian formulation for light propagation in waveguide-microresonator structures," in Proceedings of the Postconference on Quantum electronics and Laser Science (QELS '03), Trends in Optics and Photonics Series, pp. QTuL6/1-QTuL6/3, June 2003. 
[14] A. Adibi, R. K. Lee, Y. Xu, A. Yariv, and A. Scherer, "Design of photonic crystal optical waveguides with singlemode propagation in the photonic bandgap," Electronics Letters, vol. 36, no. 16, pp. 1376-1378, 2000.

[15] M. Bayindir and E. Ozbay, "Heavy photons at coupledcavity waveguide band edges in a three-dimensional photonic crystal," Physical Review B, vol. 62, no. 4, pp. R2247-R2250, 2000.

[16] M. Bayindir, B. Temelkuran, and E. Ozbay, "Propagation of photons by hopping: a waveguiding mechanism through localized coupled cavities in three-dimensional photonic crystals," Physical Review B, vol. 61, no. 18, pp. R11855-R11858, 2000.

[17] M. Bayindir, B. Temelkuran, and E. Ozbay, "Tight-binding description of the coupled defect modes in three-dimensional photonic crystals," Physical Review Letters, vol. 84, no. 10, pp. 2140-2143, 2000.

[18] Y. Xu, R. K. Lee, and A. Yariv, "Propagation and secondharmonic generation of electromagnetic waves in a coupledresonator optical waveguide," Journal of the Optical Society of America B, vol. 17, no. 3, pp. 387-400, 2000.

[19] S. Mookherjea, "Dispersion characteristics of coupledresonator optical waveguides," Optics Letters, vol. 30, no. 18, pp. 2406-2408, 2005.

[20] H. P. Seigneur, G. Gonzalez, M. N. Leuenberger, and W. V. Schoenfeld, "Dynamics of entanglement between a quantum dot spin qubit and a photon qubit inside a semiconductor high-Q nanocavity," Advances in Mathematical Physics. In press.

[21] D. Press, K. De Greve, P. L. McMahon et al., "Ultrafast optical spin echo in a single quantum dot," Nature Photonics, vol. 4, pp. 367-370, 2010.

[22] Y. Tanaka, J. Upham, T. Nagashima, T. Sugiya, T. Asano, and S. Noda, "Dynamic control of the $\mathrm{Q}$ factor in a photonic crystal nanocavity," Nature Materials, vol. 6, no. 11, pp. 862-865, 2007.

[23] S. F. Preble, Q. Xu, and M. Lipson, "Changing the colour of light in a silicon resonator," Nature Photonics, vol. 1, no. 5, pp. 293-296, 2007.

[24] PO. Dong, L. Chen, Q. Xu, and M. Lipson, "On-chip generation of high-intensity short optical pulses using dynamic microcavities," Optics Letters, vol. 34, no. 15, pp. 2315-2317, 2009.

[25] T. Tanabe, M. Notomi, H. Taniyama, and E. Kuramochi, "Dynamic release of trapped light from an ultrahigh-Q nanocavity via adiabatic frequency tuning," Physical Review Letters, vol. 102, no. 4, Article ID 043907, 2009.

[26] F. Y. Gardes, G. T. Reed, N. G. Emerson, and C. E. Png, "A sub-micron depletion-type photonic modulator in silicon on insulator," Optics Express, vol. 13, no. 22, pp. 8845-8854, 2005.

[27] T. J. Karle, Y. J. Chai, C. N. Morgan, I. H. White, and T. F. Krauss, "Observation of pulse compression in photonic crystal coupled cavity waveguides," Journal of Lightwave Technology, vol. 22, no. 2, pp. 514-519, 2004.

[28] J. Hou, H. Wu, D. S. Citrin, W. Mo, D. Gao, and Z. Zhou, "Wideband slow light in chirped slot photoniccrystal coupled waveguides," Optics Express, vol. 18, no. 10, pp. 10567-10580, 2010.

[29] D. O’Brien, M. D. Settle, T. Karle, A. Michaeli, M. Salib, and T. F. Krauss, "Coupled photonic crystal heterostructure nanocavities," Optics Express, vol. 15, no. 3, pp. 1228-1233, 2007.

[30] J. Jagerska, N. Le Thomas, V. Zabelin et al., "Experimental observation of slow mode dispersion in photonic crystal coupled-cavity waveguides," Optics Letters, vol. 34, no. 3, pp. 359-361, 2009.

[31] A. Faraon, E. Waks, D. Englund, I. Fushman, and J. Vuckovic, "Efficient photonic crystal cavity-waveguide couplers," Applied Physics Letters, vol. 90, no. 7, Article ID 073102, 2007.

[32] P. Yao and S. Hughes, "Controlled cavity QED and singlephoton emission using a photonic-crystal waveguide cavity system," Physical Review B, vol. 80, no. 16, Article ID 165128, 2009.

[33] Y. Huang and Y. W. Lu, "A defect effect to light transmission through acute bending coupled cavity waveguide in a twodimensional photonic crystal," Chinese Physics Letters, vol. 26, no. 4, Article ID 047805, 2009.

[34] E. Ozbay, M. Bayindir, I. Bulu, and E. Cubukcu, "Investigation of localized coupled-cavity modes in two-dimensional photonic bandgap structures," IEEE Journal of Quantum Electronics, vol. 38, no. 7, pp. 837-843, 2002.

[35] Y. Wang, T. Wang, and J. Liu, "Waveguide modes in coupledresonator optical waveguides," Physics Letters A, vol. 353, no. 1, pp. 101-104, 2006. 

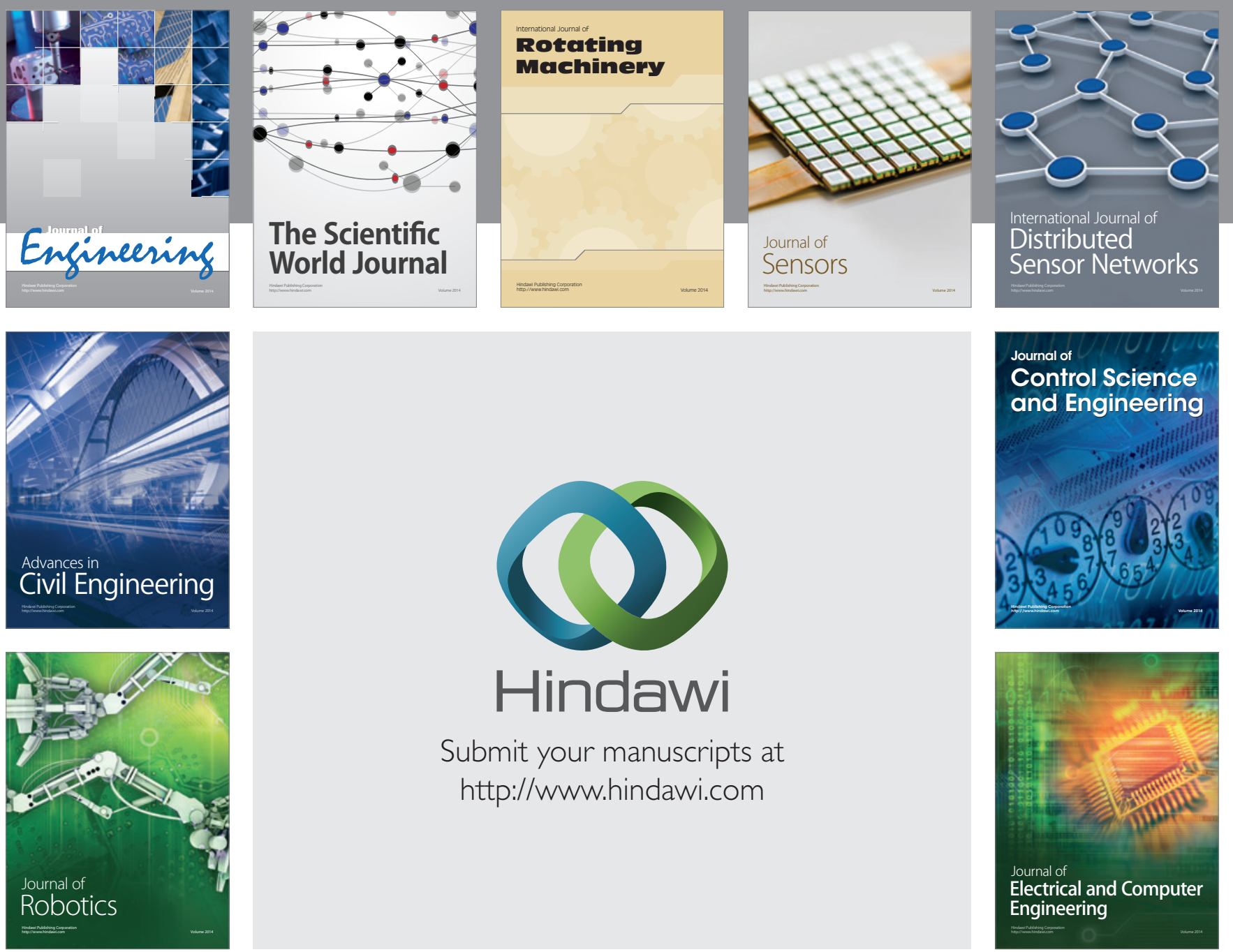

Submit your manuscripts at

http://www.hindawi.com
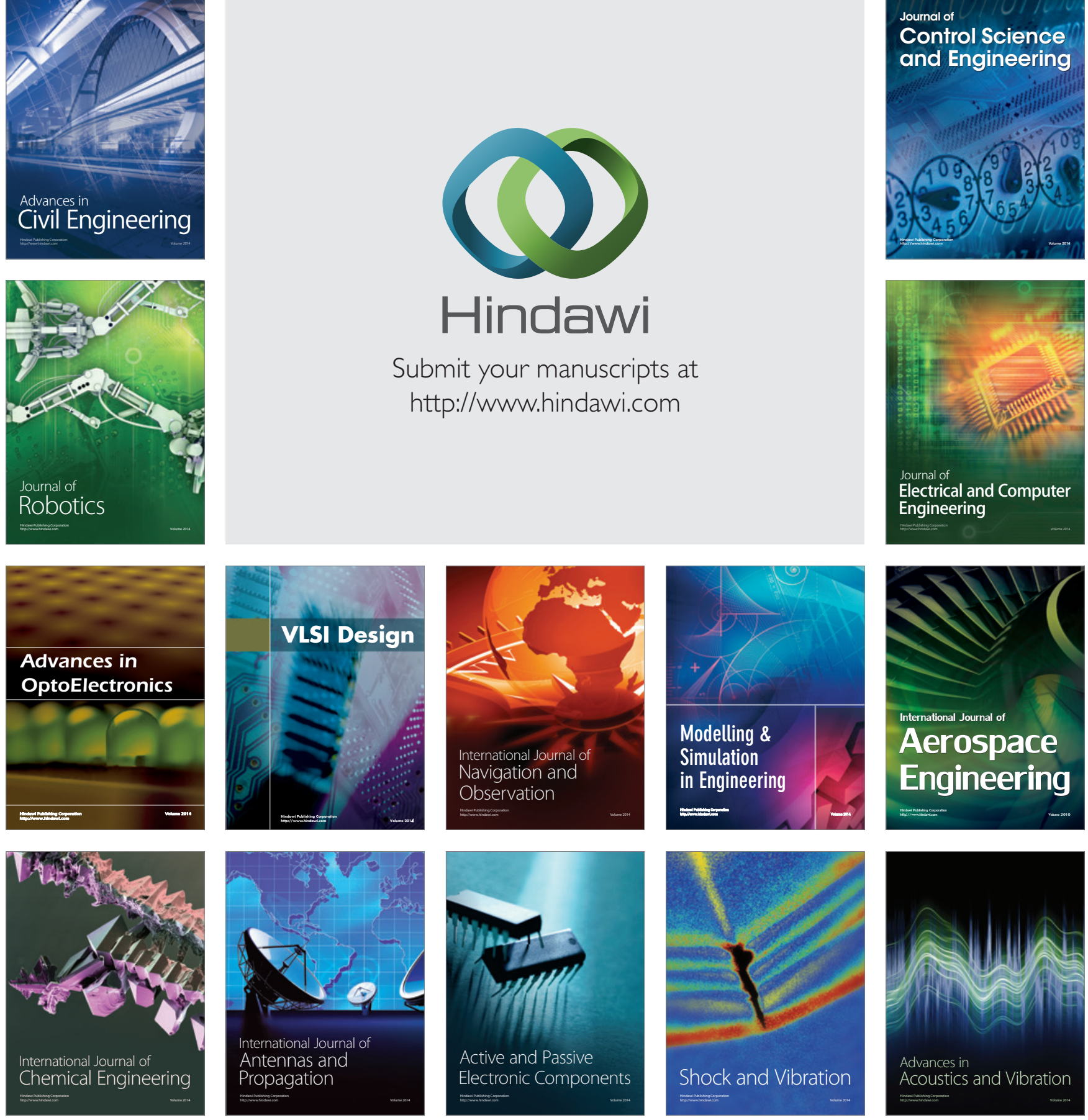